\title{
HEREDITARY FINITELY GENERATED ALGEBRAS SATISFYING A POLYNOMIAL IDENTITY
}

\author{
ELLEN E. KIRKMAN AND JAMES KUZMANOVICH
}

\begin{abstract}
If $\Lambda$ is a right and left p.p. ring which satisfies a polynomial identity and is a finitely generated algebra over its center, then $\Lambda \simeq \Gamma \times \Omega$, where $\Gamma$ is a semiprime ring having a von Neumann regular classical quotient ring which is module-finite over its center and $\Omega$ has nonzero prime radical at each of its Pierce stalks. If $\Lambda$ is right and left hereditary, then $\Gamma$ is an order over a commutative hereditary ring in the sense of [7]; the ring $\Omega$ is then a direct product of finitely many indecomposable piecewise domains.
\end{abstract}

Robson and Small [10] showed that hereditary prime PI rings are classical hereditary orders; in this paper we generalize their result to rings which are hereditary, semiprime, and finitely generated over their centers. Jøndrup [6] proved that a semiprime PI ring which is module-finite over its center and a right hereditary ring must also be a left hereditary ring; our Theorem 5 extends this result. Jøndrup [5] showed that a semiprime, right p.p., finitely generated PI algebra has a right classical quotient ring; our Corollary 4 strengthens this result, and an example is given to show that the finitely generated hypothesis cannot be dropped.

A major tool used in this paper is the Pierce sheaf representation of a ring $\Lambda$, and we now introduce the sheaf representation terminology which is used throughout. Complete details can be found in Pierce [8]. Let the Boolean ring of central idempotents of $\Lambda$ be denoted by $B(\Lambda)$ and let $X(\Lambda)$ (or just $X$ if there is no chance of confusion) denote $\operatorname{Spec}(B(\Lambda))$. The space $X$ is a totally disconnected, compact, Hausdorff space. Pierce's sheaf has $X$ as its base space and has $\Lambda_{x}=\Lambda / x \Lambda$ as the stalk at $x$ for $x \in X$. If $\lambda \in \Lambda$, then $\lambda_{x}$ denotes the image of $\lambda$ in the stalk $\Lambda_{x}$. The ring $\Lambda$ is then isomorphic to the ring of global sections of this sheaf. If $\lambda \in \Lambda$, then $\sup (\lambda)=\left\{x \in X: \lambda_{x} \neq 0_{x}\right\}$ is called the support of $\lambda$. For an open-closed subset $V$ of $X$, the central idempotent whose support is $V$ is called the associated idempotent of $V$.

Let $R$ be a commutative ring with total quotient ring $K$ and let $\Sigma$ be a central separable $K$-algebra. A module-finite $R$-subalgebra $\Lambda$ of $\Sigma$ such that $\Lambda K=\Sigma$ is called an $R$-order in $\Sigma$. The structure of a hereditary $R$-order $\Lambda$ over a commutative hereditary ring $\Lambda$ was described in [7].

The following lemma gives information about the Pierce stalks of $\Lambda$ when $\Lambda$ is semiprime and only right p.p.

Received by the editors October 22, 1980.

1980 Mathematics Subject Classification. Primary 16A14, 16A08, 16A38; Secondary 16 A16. 
LeMMA 1. Let $\Lambda$ be a PI ring which is finitely generated as an algebra over its center $R$. Suppose that $\Lambda$ is right p.p. If $x \in X(\Lambda)$ and if $J$ is a nilpotent right ideal of $\Lambda_{x}$, then $J$ can be lifted to a nilpotent right ideal I of $\Lambda$. Hence, if $\Lambda$ is semiprime, then each $\Lambda_{x}$ is prime.

Proof. By [9, Chapter V, Theorem 2.5, p. 108] $\Lambda_{x} / N\left(\Lambda_{x}\right)$ is a semiprime Goldie ring, where $N\left(\Lambda_{x}\right)$ is the prime radical of $\Lambda_{x}$, and thus $\Lambda_{x}$ has no infinite family of orthogonal idempotents. Since $\Lambda$ is right p.p, so is $\Lambda_{x}$; thus $\Lambda_{x}$ is a PWD (piecewise domain) [3]. By [3], $\Lambda_{x}$ has a triangular structure given by

$$
\Lambda_{x} \simeq\left[\begin{array}{cccc}
P_{1} & & & 0 \\
P_{21} & P_{2} & & \\
\vdots & & \ddots & \\
P_{m 1} & P_{m 2} & \cdots & P_{m}
\end{array}\right]
$$

where each diagonal ring $P_{i}$ is prime and where $N\left(\Lambda_{x}\right)$ is represented by the $P_{i j}$ blocks below the diagonal. Let $\left\{f_{1}, \ldots, f_{m}\right\}$ be a family of orthogonal idempotents of $\Lambda$ such that $\left(f_{i}\right)_{x} \Lambda_{x}\left(f_{i}\right)_{x}=P_{i}$ and $\left(f_{i}\right)_{x} \Lambda_{x}\left(f_{j}\right)_{x}=P_{i j}$. Let $\left\{g_{1}, \ldots, g_{n}\right\}$ generate $\Lambda$ as an algebra over $R$. Due to the triangular structure of $\Lambda_{x}$, we have

$$
\left(g_{k}\right)_{x}=\sum_{\substack{i=1 \\ j<i}}^{m}\left(f_{i}\right)_{x}\left(g_{k}\right)_{x}\left(f_{j}\right)_{x}
$$

for $k=1, \ldots, n$. These equations must then hold in an open-closed neighborhood $U$ of $x$. Let $e$ be the associated central idempotent of $U$. Since the equations (*) hold for each $y \in U$, and since $\left\{g_{1}, \ldots, g_{n}\right\}$ generates $\Lambda$ over $R$, it follows that $e \Lambda$ has a triangular structure given by

$$
e \Lambda \simeq\left[\begin{array}{ccc}
\left(e f_{1}\right) e \Lambda\left(e f_{1}\right) & & 0 \\
\vdots & \ddots & \\
\left(e f_{m}\right) e \Lambda\left(e f_{1}\right) & \cdots & \left(e f_{n}\right) e \Lambda\left(e f_{n}\right)
\end{array}\right]
$$

Let

$$
M=e\left[\sum_{\substack{i=2 \\ j \leq i}}^{m} f_{i} \Lambda f_{j}\right]=\sum_{\substack{i=2 \\ j \leq i}}^{m}\left(e f_{i}\right) e \Lambda\left(e f_{j}\right) .
$$

It is clear from the triangular structure of $e \Lambda$ that $M$ is an ideal of $\Lambda$ which is nilpotent of index at most $m$. Let $I=\left\{\lambda \in M: \lambda_{x} \in J\right\}$. It is easily verified that $I$ is a nilpotent right ideal for which $I_{x}=J$.

The ideal $M$ is a lifting of $N\left(\Lambda_{x}\right)$ to a nilpotent ideal of $\Lambda$; it follows that if $\Lambda$ is semiprime, then $\Lambda_{x}$ is semiprime. The ring $\Lambda_{x}$ is thus prime, for a semiprime PWD is a direct sum of prime rings, but $\Lambda_{x}$ must be indecomposable since $\Lambda$ is a finitely generated algebra over $\boldsymbol{R}$. 
Bergman [1] characterized the commutative p.p. rings as being those commutative rings whose Pierce stalks were all integral domains and in which elements had open-closed support. The following is a generalization of his result. The two-sided p.p. assumption is needed in the hypothesis.

Proposition 2. Let $\Lambda$ be a two-sided p.p. ring which is $P I$ and which is a finitely generated algebra over its center $R$. The elements of $\Lambda$ have open-closed support.

Proof. Let $\lambda \in \Lambda$. Since $\Lambda$ is p.p. there are idempotents $e$ and $f$ in $\Lambda$ such that $\operatorname{rann}(\lambda)=\operatorname{rann}(e)$ and $\operatorname{lann}(\lambda)=\operatorname{lann}(f)$. It is not difficult to see that $\sup (\lambda)=$ $\sup (e)=\sup (f)$ and $f \lambda=\lambda=\lambda e$.

Take $0 \neq \sigma \in \Lambda$. Supports are always closed; therefore, it will be sufficient to show that $\sup (\sigma)$ is open. Let $x \in X(\Lambda)$ be an element of $\sup (\sigma)$. Define $I=$ $\left\{u \in \Lambda_{x}: u=\lambda_{x}\right.$ for $\lambda \in \Lambda$ with $\left.\sup (\lambda) \subset \sup (\sigma)\right\}$. The ideal $I$ is a two-sided ideal of $\Lambda_{x}$, and since $x \in \sup (\sigma), I$ is nonzero. By the above observation, for every $u \in I$ with $u=\lambda_{x}$, there exist idempotents $e=e(\lambda), f=f(\lambda)$ in $\Lambda$ such that $f \lambda=\lambda=\lambda e$ and $e_{x} \in I, f_{x} \in I$. Thus if $J$ is any ideal of $\Lambda_{x}$, then $I \cap J=I J=$ $J I$.

Let $N$ be the prime radical of $\Lambda_{x}$. As in Lemma $1, \bar{\Lambda}_{x}=\Lambda_{x} / N$ (use bars to denote images in $\bar{\Lambda}_{x}$ ) is a semiprime Goldie ring; $\bar{\Lambda}_{x}$ is also p.p. so that it is a direct product of finitely many prime rings. Let $S$ be one such prime factor in which $I$ has nonzero image $\bar{I}$. In this case $\bar{I}$ contains a regular element $\bar{b}$ of $S$, since $S$ is a prime Goldie ring. Let $e$ be an idempotent in $I$ such that $e b=b$. Clearly $\overline{e b}=\bar{b}$, which by the regularity of $\bar{b}$ implies that $\bar{e}=1_{s}$ and $\bar{I}=S$. This holds for each such prime factor of $\bar{\Lambda}_{x}$ from which it follows that there is an idempotent $h$ in $I$ which is central in $\bar{\Lambda}_{x}$ for which $I=h \Lambda_{x}+(I \cap N)$. Since $N$ is nilpotent, $I=h \Lambda_{x}+$ $(I \cap N)=h \Lambda_{x}+I N=h \Lambda_{x}$. Similarly, there is an idempotent $g$ in $I$ for which $I=\Lambda_{x} g$. It then follows that $h=h g=g$ is a central idempotent of $\Lambda_{x}$. Since $\Lambda$ is a finitely generated algebra, $\Lambda_{x}$ is indecomposable and $h=1_{x}$. Lift $h$ to an idempotent $f$ of $\Lambda$ such that $f_{x}=h=1_{x}$ and $\sup (f) \subset \sup (\sigma)$. There is an openclosed neighborhood of $x$ with associated central idempotent $k$ such that $1_{y}=f_{y}$ for all $y$ in the neighborhood. Then $f k$ is a central idempotent of $\Lambda$ with $x \in \sup (f k) \subset \sup (f) \subset \sup (\sigma)$. Thus $\sup (f k)$ is an open neighborhood of $x$ contained in $\sup (\sigma)$. Consequently $\sup (\sigma)$ is open as desired.

The following theorem is a generalization of results in [2].

THEOREM 3. Let $\Lambda$ be a PI ring which is a finitely generated algebra over its center R.

(i) If $\Lambda$ is left and right p.p., then $\Lambda=\Gamma \times \Omega$ where $\Gamma$ is a semiprime ring while the ring $\Omega$ has nonzero prime radical at each of its Pierce stalks. The ring $\Gamma$ has a regular total quotient ring which is module-finite over its center and is obtained from $\Gamma$ by inverting regular central elements.

(ii) If in addition $\Lambda$ is hereditary, then the semiprime ring $\Gamma$ is module-finite over its center (and hence its center is hereditary and it is an order in the sense of [7]) and the ring $\Omega$ is the direct product of finitely many indecomposable piecewise domains with nonzero prime radical. 
Proof. (i) It is sufficient to show that the set $S=\left\{x \in X: \Lambda_{x}\right.$ is prime $\}$ is open-closed. Let $\Sigma$ be the ring obtained from $\Lambda$ by inverting the regular central elements. It is easy to see that $X(\Lambda)=X(\Sigma)$ and that $K$ is the center of $\Sigma$ where $K$ is the total quotient ring of $R$.

Take $x \in S$. The ring $\Lambda_{x}$ is a prime PI ring; consequently, an extension of Posner's theorem [9, p. 175] implies that $\Sigma_{x}$ is the simple Artinian classical quotient ring of $\Lambda_{x}$. Hence $S=\left\{x \in X: \Sigma_{x}\right.$ is a central separable $K_{x}$-algebra $\}$. For a finitely generated algebra the property of being module-finite over its center can be expressed in a finite number of equations. Therefore, if $x \in S$, then there exists an open-closed neighborhood $U$ of $x$ such that $\Sigma_{y}$ is module-finite over $K_{y}$ for all $y \in U$. The separability of a module-finite algebra can also be expressed in terms of a finite number of equations; hence there is an open-closed neighborhood $V \subset U$ of $x$ for which $\Sigma_{y}$ is central separable for all $y \in V$. This shows that $S$ is an open set.

Conversely, suppose that $x \notin S$; that is, suppose that $\Lambda_{x}$ is not prime (and hence not semiprime as noted above). Let $J$ be a nonzero nilpotent ideal of $\Lambda_{x}$. By Lemma 1 there exists a nilpotent ideal $I$ of $\Lambda$ such that $I_{x}=J$. Applying Proposition 2 yields that supports of elements are open-closed and hence that there exists an open-closed neighborhood $U$ of $x$ such that $I_{y}$ is a nonzero nilpotent ideal of $\Lambda_{y}$ for all $y \in U$. This shows that $\Lambda_{y}$ is not prime for each such $y$ and that $U \subset X-S$. Hence $X-S$ is open and $S$ is closed.

Let $e$ be the central idempotent of $\Lambda$ for which $\Gamma=e \Lambda$. The ring $e \Sigma$ is regular since $(e \Sigma)_{x}$ is simple Artinian for each $x \in X(\Gamma)$. It follows that $e \Sigma$ is the total quotient ring of $\Gamma$, for it is regular and is obtained by inverting regular central elements of $\Lambda$. A standard sheaf argument shows that $e \Sigma$ is module-finite over its center.

(ii) If $x \in S=X(\Gamma)$, then $\Gamma_{x}$ is a hereditary prime PI ring if $\Lambda$ is hereditary. Hence by [10] each $\Gamma_{x}$ is a classical order over its center which is a Dedekind domain. The property of being module-finite over its center can be expressed in finitely many equations for a finitely generated algebra. A standard sheaf argument then yields that $\Gamma$ is module-finite over its center.

To show that $\Omega$ is a direct product of finitely many indecomposable rings it will be sufficient to show that $X(\Omega)$ is finite. Suppose that $X(\Omega)$ is infinite. In this case $X(\Omega)$ has a limit point $y$ since $X(\Omega)$ is compact. Let $M=\left\{w \in \Omega: w_{y}=0_{y}\right\}$. Let $u \in \Omega$ such that $u_{y} \neq 0_{y}$ and $\left(u_{y} \Omega_{y}\right)^{2}=0_{y}$. Let $I=M+u \Omega$. Observe that since $X(\Omega)$ is Hausdorff, $I_{x}=\Omega_{x}$ if $x \neq y$ and $I_{y}=u_{y} \Omega_{y}$. A contradiction will be obtained by showing that the ideal $I$ cannot be projective. If $I$ were projective, then $I=\bigoplus J_{\alpha}$ where each $J_{\alpha}$ is a finitely generated right ideal $\Omega$. There is no loss of generality in assuming that exactly one $J_{\alpha}$, say $J_{1}$, is nonzero at $y$; hence $\left(J_{1}\right)_{y}=$ $\left(u_{y} \Omega_{y}\right)$. By Lemma $1\left(u_{y} \Omega_{y}\right)$ can be lifted to a nilpotent right ideal of $\Omega$. The right ideal $J_{1}$ is finitely generated, so there is an open-closed neighborhood $U$ of $y$ such that $\left(J_{1}\right)_{z}$ is nilpotent for all $z \in U$. By Proposition 2 supports are open-closed so there is no loss of generality in assuming that $\left(J_{1}\right)_{z} \neq 0_{z}$ for all $z \in U$. Since $y$ is a limit point, there exists $x \in U, x \neq y$. The ideal $I_{x}=\left(\bigoplus J_{\alpha}\right)_{x}=\bigoplus\left(J_{\alpha}\right)_{x}=\Omega_{x}$. 
The nilpotent right ideal $\left(J_{1}\right)_{x}$ is superfluous in $\Omega_{x}$, which implies that $\bigoplus_{\alpha \neq 1}\left(J_{\alpha}\right)_{x}$ $=\Omega_{x}$. However, $0_{x} \neq\left(J_{1}\right)_{x}=\left(J_{1}\right)_{x} \cap\left(\bigoplus_{\alpha \neq 1}\left(J_{\alpha}\right)_{x}\right)$. This contradicts the fact that the sum of the family $\left\{J_{\alpha}\right\}$ is direct. Hence $I$ is not projective and $X(\Omega)$ is finite.

It was noted in the proof of Lemma 1 that each $\Omega_{x}$ is a PWD.

COROllary 4. Let $\Lambda$ be a semiprime PI ring which is a finitely generated algebra over its center $R$ and let $K$ be the total quotient ring of $R$. If $\Lambda$ is right p.p., then $\Lambda$ has a regular classical quotient ring $\Sigma$ which is module-finite over its center $K$ and is obtained from $\Lambda$ by inverting regular central elements.

Proof. The proof of Theorem 3 uses the two-sided p.p. hypothesis only to obtain the ring decomposition; hence, if $\Lambda$ is semiprime the assumption of right p.p. is sufficient to obtain the properties of $\Sigma$.

Theorem 3 can be interpreted as saying that a hereditary, finitely generated, PI algebra is at most a PWD ring direct summand away from being module-finite over its center. Indeed, such PWD's need not be module-finite algebras; an example of such is the ring

$$
\Omega=\left[\begin{array}{ccc}
F & 0 & 0 \\
F[x] & F[x] & 0 \\
F[x] & F[x] & F
\end{array}\right]
$$

The ring $\Omega$ above does not have a classical quotient ring which shows that the semiprime hypothesis cannot be dropped in Corollary 4.

The finitely generated hypothesis also cannot be dropped in Corollary 4 . Let

$$
\Gamma=\oplus\left[\begin{array}{lll}
F(x) & F(x) & F(x) \\
F(x) & F(x) & F(x) \\
F(x) & F(x) & F(x)
\end{array}\right]+\operatorname{long}\left[\begin{array}{ccc}
F & 0 & 0 \\
F[x] & F[x] & 0 \\
F[x] & F[x] & F
\end{array}\right]
$$

where $x$ is an indeterminant over the field $F$ and "long" denotes a constant sequence of the indicated form. The ring $\Gamma$ is a hereditary semiprime PI ring, but it does not possess a classical quotient ring. This answers a question of Jøndrup [5].

Finally, it was indicated in [2, Example 2.3] that the ring decomposition of Theorem 3 cannot be obtained under the hypothesis that $\Lambda$ is hereditary on only one side, even when $\Lambda$ is module-finite over $R$. This example [2, Example 2.3] also shows that the semiprime hypothesis is needed in the following theorem.

THEOREM 5. Let $\Lambda$ be a semiprime PI ring which is a finitely generated algebra over its center $R$.

(i) If $\Lambda$ is right p.p., then $\Lambda$ is left p.p.

(ii) If $\Lambda$ is right semihereditary, then $\Lambda$ is left semihereditary.

(iii) If $\Lambda$ is right hereditary, then $\Lambda$ is left hereditary.

Proof. (i) Take $\lambda \in \Lambda$. Let $\Sigma$ be the regular classical quotient ring of $\Lambda$ which exists by Corollary 4. Each $\Lambda_{x}$ is a prime Goldie ring and hence cannot contain an infinite family of orthogonal idempotents. It follows from a result of Small [11] that each $\Lambda_{x}$ is left p.p.; hence, for each $x \in X$ there exists an idempotent $f(x)$ in $\Lambda$ 
such that $\operatorname{lann}_{\Lambda_{x}}\left(\lambda_{x}\right)=\Lambda_{x} f(x)_{x}$. The ring $\Sigma$ is regular, whence lann $\Sigma(\lambda)=\Sigma e$ for an idempotent $e$ of $\Sigma$. Since $e=b^{-1} a$ where $a \in \operatorname{lann}_{\Lambda}(\lambda)$ and $b$ is a regular central element of $\Lambda$, the equations

$$
a_{x} f(x)_{x}=a_{x}, \quad f(x)_{x} \lambda_{x}=0_{x}, \quad\left(f(x)_{x}\right)^{2}=f(x)_{x}
$$

hold for each $x \in X$. The equations (**) can be lifted and patched in the standard sheaf theoretic manner to yield an idempotent $f$ of $\Lambda$ for which $e f=e$ and $f \lambda=0$. Clearly $\Lambda f \subset \operatorname{lann}_{\Lambda}(\lambda)$. Conversely, let $\rho \in \operatorname{lann}_{\Lambda}(\lambda)$. Since $\operatorname{lann}_{\Lambda}(\lambda) \subset \operatorname{lann}_{\Sigma}(\lambda)=$ $\Sigma e$, we have $\rho f=\rho e f=\rho e=\rho$. Thus $\operatorname{lann}_{\Lambda}(\lambda)=\Lambda f$ and $\Lambda f$ is projective. This shows that $\Lambda$ is left p.p.

(ii) If $\Lambda$ is right semihereditary, then $M_{n}(\Lambda)$, the $n \times n$ matrix ring over $\Lambda$, is right p.p. for any $n \geqslant 1$. All the hypotheses of (i) apply to $M_{n}(\Lambda)$, so that (i) implies that $M_{n}(\Lambda)$ is left p.p. for $n \geqslant 1$; that is, $\Lambda$ is left semihereditary.

(iii) If $\Lambda$ is right hereditary, then each $\Lambda_{x}$ is a right hereditary prime PI ring, and by [10] each $\Lambda_{x}$ is then module-finite over its center. The ring $\Lambda$ is hereditary by [4]. Hence $\Lambda$ is an order over a hereditary ring in the sense of [7]. By [7, Theorem 3.5] $\Lambda$ is left hereditary.

As a corollary to Theorem 3 and Theorem 5 we have the following. It can be viewed as a generalization of Robson and Small [10].

COROLlaRY 6. Let $\Lambda$ be a right hereditary semiprime PI ring which is a finitely generated algebra over its center. Then $\Lambda$ is a hereditary order over a commutative hereditary ring.

Theorem 5 is not valid for semiprime PI rings which are not finitely generated algebras over their centers. For a field $F$ denote elements of $T=(\Pi F) \oplus$ $\left(\Pi M_{2}(F)\right)$ as ordered pairs $(u, v)$ with $u \in \Pi F$ and $v \in \Pi M_{2}(F)$. Let $\Lambda$ be the subring of $T$ generated by $(\bigoplus F) \oplus\left(\bigoplus M_{2}(F)\right)$ and all elements of the form (long $a$, long $\left.\left[\begin{array}{ll}a & 0 \\ b & c\end{array}\right]\right)$. The ring $\Lambda$ is a left hereditary semiprime PI ring which is not even right p.p.

\section{REFERENCES}

1. G. Bergman, Hereditary commutative rings and centres of hereditary rings, Proc. London Math. Soc. (3) 23 (1971), 214-236.

2. J. Fuelberth, E. Kirkman and J. Kuzmanovich, Hereditary module-finite algebras, J. London Math. Soc. (2) 19 (1979), 268-276.

3. R. Gordon and L. W. Small, Piecewise domains, J. Algebra 23 (1972), 553-564.

4. S. Jondrup, The centre of a right hereditary ring, J. London Math. Soc. (2) 15 (1977), 211-212.

5. ___ Rings of quotients of some semiprime PI rings, Comm. Algebra 4(1976), 51-75.

6. Homological dimension of some P.I. rings, Preprint Series, no. 16, Kobenhavns Universitet, Kobenhavns, 1979.

7. E. Kirkman and J. Kuzmanovich, Orders over hereditary rings, J. Algebra 55 (1978), 1-27.

8. R. S. Pierce, Modules over commutative regular rings, Mem. Amer. Math. Soc., no. 70 (1967).

9. C. Procesi, Rings with polynomial identities, Dekker, New York, 1973.

10. J. C. Robson and L. W. Small, Hereditary prime P.I. rings are classical hereditary orders, J. London Math. Soc. (2) 8 (1974), 499-503. MR 50 \#2236.

11. L. W. Small, Semihereditary rings, Bull. Amer. Math. Soc. 73 (1967), 656-658. MR 35 \#2926.

Department of Mathematics, Wake Forest Universtty, Winston-Salem, North Carolina 27109 